\title{
The study of risk in pulmonary arterial hypertension
}

\author{
Lewis J. Rubin*, Gerald Simonneau\#, David Badesch", Nazzareno Galiè+, \\ Marc Humbert ${ }^{\#}$, Anne Keogh ${ }^{\S}$, Joseph Massaro ${ }^{f}$, Marco Matucci Cerinic**,\#\#, \\ Olivier Sitbon ${ }^{\#}$ and Steven Kymes"
}

\begin{abstract}
A growing body of published evidence exists on the risk factors for disease progression in pulmonary arterial hypertension (PAH). The Scientific Steering Committee for the Study of Risk in PAH was established to bring together leading clinical and statistical experts in PAH and risk modelling, for the purpose of advancing the understanding of the risk of development and progression of PAH. Herein, we discuss the impact of this information on three key areas: 1) clinical decision-making; 2) policy and reimbursement; and 3) future trials and research.
\end{abstract}

KEYWORDS: Economics, prognosis, pulmonary arterial hypertension, quality of life, risk, survival

\section{PROGNOSIS FOR SURVIVAL}

Despite the limitations in comparing data across trials and from different treatment eras, survival for pulmonary arterial hypertension $(\mathrm{PAH})$ does seem to have improved in the modern era $[1,2]$ versus historical survival from the National Institutes of Health (NIH) registry [3]. However, outcomes are still unacceptably low and treatment strategies need to evolve in order to improve survival.

Outcomes in PAH vary for different aetiologies, with systemic sclerosis (SSc)-associated PAH patients having among the worst survival and idiopathic PAH patients who respond to vasoreactivity testing the best survival [4-6]. Survival is also worse for incident (newly diagnosed) versus prevalent patients [1]

High-risk patients, such as those with SSc, may be screened for PAH. Earlier detection and earlier intervention may lead to improved outcome [7].

Age, sex, 6-min walking distance (6MWD) and cardiac output/cardiac index are commonly identified as having a strong prognostic loading. Different variables emerge as significant from different data sets, e.g. in the Bologna PAH experience, age and sex are important risk factors in some clinical subgroups but not all, whilst cardiac output is not identified as a risk factor, in contrast with other analyses (such as the French registry).

There are a number of considerations when analysing predictive factors from datasets, including:
1) statistical power sample size; 2) categorical (e.g. World Health Organization-functional class (WHOFC)) versus continuous (e.g. 6MWD) variables; 3) subgroup-specific differences (e.g. age and sex); and 4) lack of variability (e.g. majority of subjects are WHO-FC III at baseline).

Since reduced cardiac output is frequently predictive of outcome, the Scientific Steering Committee for the Study of Risk in PAH (hereafter referred to as the group) considered the impact of heart rate, stroke volume and pulse pressure. Data from the sildenafil PAH development programme indicate that heart rate at baseline, and systemic pulse pressure at follow-up, were predictive of outcome [8]. It is of interest to perform similar analyses in other real-world, registry and clinical trial datasets.

The group noted the apparent increasing age of PAH patients, which is $>50$ yrs in recent registries such as the French [1], REVEAL (Registry to Evaluate Early and Long-Term Pulmonary Arterial Hypertension Disease Management) [2] and CompERA [9] versus 35 yrs in the original $\mathrm{NIH}$ registry (table 1) [10]. This raises a number of questions. 1) Is $\mathrm{PAH}$ the same disease in elderly versus younger patients? 2) How do we interpret efficacy data from clinical trials, where the mean age is $\sim 50$ yrs, for older subjects? 3) Are older, often geriatric, patients as responsive to therapy (especially to vasodilators) as younger patients? 4) Is there a risk of targeted therapies

\section{AFFILIATIONS}

* School of Medicine, University of California San Diego, La Jolla, CA, - University of Colorado Health Sciences Center, Denver, CO, ${ }^{f}$ School of Public Health, Boston University, Boston, MA, and

" "Dept of Ophthalmology, Washington University School of

Medicine, St Louis, MO, USA

\# Service de Pneumologie, University of South Paris, Hôpital Bicêtre, Le Kremlin-Bicêtre, France.

${ }^{+}$Dept of Cardiology, University of

Bologna, Bologna, and

**Dept of Biomedicine, Division of Rheumatology, AOUC, and

\#\# Dept of Medicine, Denothe centre, University of Florence, Florence, Italy.

${ }^{\S}$ Heart and Lung Transplant Unit, St Vincent's Public Hospital, Sydney, Australia.

\section{CORRESPONDENCE}

L.J. Rubin

School of Medicine

University of California San Diego 9500 Gilman Dr.

La Jolla

CA 92093

USA

E-mail: ljr@lewisrubinmd.com

Received:

June 082012

Accepted:

June 192012

\section{PROVENANCE}

Publication of this peer-reviewed article was supported by Pfizer, UK (article sponsor, European Respiratory Review issue 125).

European Respiratory Review Print ISSN 0905-9180

Online ISSN 1600-0617 
TABLE 1 Patient characteristics in the National Institutes of Health $(\mathrm{NIH})$, French and REVEAL registries

\begin{tabular}{|c|c|c|c|c|}
\hline Parameter & NIH [10] & French [1] & REVEAL [2] & CompERA [9] \\
\hline Year & $1981-1985$ & 2002-2003 & 2006-2007 & 2007-2009 (data collection ongoing) \\
\hline Country & USA & France & USA & $\begin{array}{c}6 \text { EU countries: Germany, UK, Belgium, } \\
\text { Netherlands, Italy and Ireland }\end{array}$ \\
\hline Inclusion criteria & $\mathrm{PPH}$ & PAH & $\mathrm{PAH}$ & $\begin{array}{l}\text { Newly initiated on PAH therapy } \\
\text { (PAH 74\%; PH 26\%) }\end{array}$ \\
\hline Patients & 187 & 674 & 2716 & 1008 \\
\hline Age yrs & $36 \pm 15$ & $50 \pm 15$ & $50 \pm 17$ & 64 \\
\hline Time since diagnosis months & 24.4 & 27 & 39 & Not given \\
\hline \multicolumn{5}{|l|}{ WHO-FC \% } \\
\hline$\|$ & 29 & 24 & 37.8 & 9 \\
\hline III/IV & 71 & 75 & 53.7 & 91 \\
\hline
\end{tabular}

Data are presented as $n$ or mean \pm SD, unless otherwise stated. REVEAL: Registry to Evaluate Early and Long-Term Pulmonary Arterial Hypertension Disease Management; WHO-FC: World Health Organization-functional class; PPH: primary pulmonary hypertension (PH); PAH: pulmonary arterial hypertension; EU: European Union.

being considered "end-of-life" drugs by reimbursement bodies? 5) Is the haemodynamic definition of pulmonary hypertension (PH), using an upper limit of $15 \mathrm{mmHg}$ for pulmonary capillary wedge pressure $(P \mathrm{pcw})$, still valid to define pre- versus postcapillary $\mathrm{PH}$, or are there differences, for example, between subjects with $P_{\mathrm{pcw}}<12 \mathrm{mmHg}$ versus $12-15 \mathrm{mmHg}$ ?

An analysis of the impact of baseline Ppcw on outcome from the sildenafil clinical trial programme found that there were differences in some end-points between patients with $P$ pcw $<12 \mathrm{mmHg}$ versus $>12 \mathrm{mmHg}$ [11]. This haemodynamic classification of PAH will probably be debated at the 5th Word PH Symposium in Nice, France, in February 2013.

Male sex is a risk factor for poor prognosis. In the French registry the female/male ratio is 1.7 , but this increases to 4 in the REVEAL registry [1, 2]. What are the reasons for these regional differences in sex? Do these differences exist in other datasets, such as clinical trials and other registries?

\section{RISK ASSESSMENT}

Prognostic equations have been developed from existing datasets, including the French and REVEAL registries $[2,4]$. These equations describe the survival of the specific population. However, additional value could be obtained through development of a tool, e.g. risk calculator, from such predictive models to: 1) simplify the complex reality of individual PAH patients; 2 ) aid non-expert clinicians; 3) stimulate expert referral, especially for the most severe patients for whom complex treatment options are indicated; and 4) determine when to select a particular intervention, e.g. i.v. epoprostenol or listing for transplantation.

The REVEAL risk factors were given a numerical score based on severity, allowing for the creation of a risk calculator that can be used to derive a severity score and risk for poor outcome based on the presence or absence of the criteria [5]. A total of 19 parameters were included (10 from aetiology and physical examination and nine from diagnostic tests), all of which have a sound basis.

The REVEAL model and risk calculator have been successfully validated in separate US PAH populations, both incident and prevalent [5], and further validation is planned. Similarly, the French registry equation had good reproducibly when tested in a Chinese cohort [12] and the REVEAL cohort [13].

\section{DYNAMIC RISK ASSESSMENT}

Baseline clinical values clearly have importance in risk assessment. However, their use is limited as prognosis may change over time with interventions and as the clinical picture changes. There is a need for assessment at multiple follow-up time-points (dynamic assessment) to evaluate the impact of timedependent variables on clinical outcomes. A single centre study from Hannover, Germany, evaluated the prognostic impact of follow-up assessment in 109 incident PAH patients [14]. After identifying prognostic factors for survival by multivariate analysis, it was feasible to stratify patients' outcomes following the European Society of Cardiology/European Respiratory Society guidelines criteria for risk assessment (fig. 1). Despite a small sample size, the study is hypothesis generating and demonstrates the need to evaluate a broad number of clinical

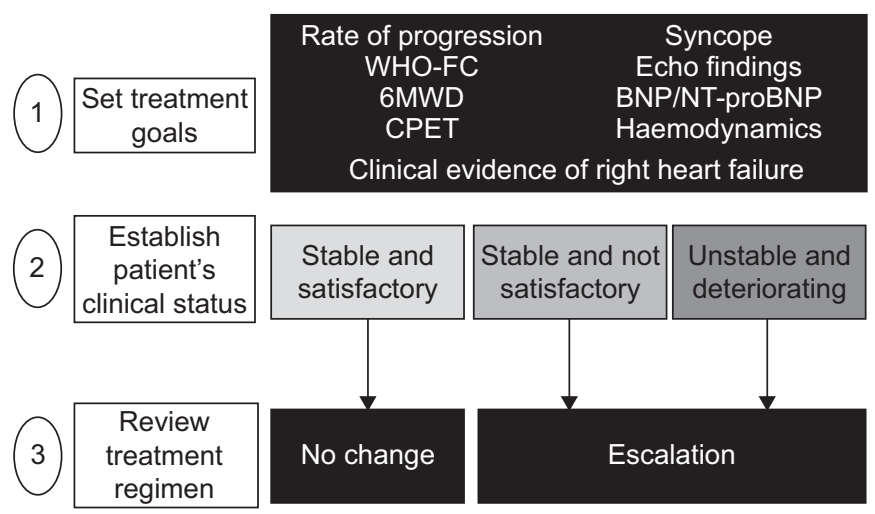

FIGURE 1. Risk assessment and treat-to-target approach for pulmonary arterial hypertension. WHO-FC: World Health Organization-functional class; 6MWD: 6-min walking distance; CPET: cardiopulmonary exercise testing; Echo: echocardiography; BNP: brain natriuretic peptide; NT-proBNP: N-terminal-pro-brain natriuretic peptide. Adapted from [15] with permission from the publisher. 
factors covering all pathophysiological aspects of the disease in order to regularly re-assess treatment goals.

It may be feasible to use prospective registries to assess timedependent prognostic variables. We can consider that there are three types of registries, all with strengths and limitations (table 2).

A novel prospective study is ongoing in France, the EFORT study (Evaluation of Prognostic FactORs and Treatment Goals in PAH) [16]. The study is enrolling newly diagnosed patients with idiopathic, heritable and anorexigen-associated PAH within the French PAH network. Regular clinical assessment and follow-up (at baseline, 3-4 months after treatment initiation or treatment change, and annually) for 3 yrs will identify prognostic factors for survival, and determine treatment strategies associated with improved outcome.

In order to increase the sample size and generalisability of the data, it was suggested to explore options to open up the study to expert centres from other countries.

From the EFORT study, it may be feasible to stratify subjects as low, medium or high risk, despite the applied treatment strategy. Subsequent research could evaluate whether changing the treatment strategy based on risk can impact patient outcome. Whilst the group acknowledges such studies may not provide the final answer, they can help to generate new questions and eventually lead to improvement in treatment guidelines and outcomes for PAH patients.

\section{TREATMENT STRATEGIES}

Current risk equations and calculators have been developed from cohorts of subjects who have received interventions from a treating clinician. Therefore, how do we interpret the risk score for a particular patient and adjust the treatment strategy accordingly?

In patients with poor prognosis at baseline, combination therapy is an option [15]. To date, one study has demonstrated improved outcome with combination versus monotherapy in PAH [17]. Ongoing trials (e.g. AMBITION [18], COMPASS-2 [19] and A1481243 [20]) will provide additional data on how treatment strategy impacts outcome (e.g. goal-oriented versus up-front combination therapy). In a small, open-label study, up-front triple therapy with bosentan, sildenafil and i.v. epoprostenol in severe patients had a dramatic impact on

\begin{tabular}{|c|c|}
\hline TABLE 2 & $\begin{array}{l}\text { Types of prospective registries for analysis of } \\
\text { time dependent prognostic variables }\end{array}$ \\
\hline \multicolumn{2}{|c|}{ Non-selective registries } \\
\hline \multicolumn{2}{|c|}{ Pros: epidemiology (prevalence) and clinical characteristics } \\
\hline \multicolumn{2}{|c|}{ Cons: survival analysis and prognosis } \\
\hline \multicolumn{2}{|c|}{ Registries of newly diagnosed patients } \\
\hline \multicolumn{2}{|c|}{$\begin{array}{l}\text { Pros: epidemiology (incidence), survival analysis, prognosis and risk } \\
\text { equation from baseline variables }\end{array}$} \\
\hline \multicolumn{2}{|c|}{ Cons: assessment of time-dependent variables and treatment goals } \\
\hline \multicolumn{2}{|c|}{ Interventional registries } \\
\hline Pros: asses & ment of time-dependent variables and treatment goals \\
\hline
\end{tabular}

haemodynamics and clinical outcomes when compared with previous experience on mono- or dual therapy [21].

The outcome of the AMBITION trial is eagerly awaited; if positive, it could lead to a change in the treatment paradigm to initiate patients on oral combination therapy. However, risk assessment will remain crucial to guide treatment strategy. It is important to remember that transplantation is a therapeutic option for the most severe patients, which further highlights the importance of referral to expert centres.

\section{HEALTH ECONOMICS, TECHNOLOGY ASSESSMENT AND PAH}

Economic evaluation is a family of methods and techniques that have been employed by health policy makers in many developed nations to assist them in making decisions concerning the adoption of new healthcare technologies. Among the most commonly employed techniques are cost-minimisation and costutility analysis that are designed to determine how the value created by the new treatment compares to the cost of its adoption. In cost-minimisation analyses, only the cost of treatment is concerned; therefore, the decision maker is left to assume that the treatment efficacy is equal among the therapies. In cost-utility analysis, this assumption is relaxed and efficacy is measured by its impact on quality of life measures of function [22].

However, it has not been necessary for companies developing drugs for palliative treatment to develop extensive dossiers using all of the tools of economic evaluation. Often it is only necessary for them to provide evidence of clinical efficacy and document budget impact for health authorities being petitioned. The reason for this is the special status of PAH as an orphan disease. This implies that while treatment cost per case is high, the low prevalence and high mortality result in a total monetary burden representing a relatively tiny portion of the total healthcare budget.

The group recognised that this status makes it even more important for clinicians to be aware of the balance between the efficacy of medications and their cost in choosing the optimal treatment for patients. The largest contributor to the cost of care associated with PAH is the cost of pharmaceutical treatment, and this varies dramatically ranging from more than US $\$ 9,000$ (sildenafil) to more than US\$60,000 (iloprost) annually (J.R. Edler, Center for Economic Evaluation in Medicine, Washington University School of Medicine, St. Louis, MO, USA; personal communication). When we couple this with the results of a 2009 report by CHEN et al. [23], which found good evidence for the cost-effectiveness of bosentan and sildenafil (at current threshold standards), clinicians in most countries can be confident that they can match treatments to their patient's needs and still make good use of societal resources.

However, this may change in the future with the advent of generic drugs, novel therapies that promise to be even more expensive than current ones, and a worsening global macroeconomic environment. In the UK and Australia, for example, there are already limitations on access to therapies for less severe patients (WHO-FC II), as well as on the use of combination therapy. The group considered including evaluation of costs into PAH clinical trials. This will probably become an increasingly important requirement for successful health technology assessment and application for reimbursement. 
In addition to measuring the cost of care in trials, the group acknowledged that measuring the benefit of treatments for the $\mathrm{PAH}$ patient using quality of life measures appropriate for economic evaluation is a challenge that remains to be addressed. Patient-reported outcomes data have been collected in PAH trials, mostly using the 36-item Short-Form Health Survey and the EuroQoL-5D. However, the responsiveness of these measures to clinically significant changes in disease status has not been well established. Establishing the validity of these measures in $\mathrm{PAH}$ would be a useful area for future research. If they are found to be inadequate, there may be a need for a new instrument to support decision making by health policy makers [24-26].

\section{CONCLUSION}

$\mathrm{PAH}$ is rare condition, and whilst improvements in outcome have been achieved, survival remains poor. Data should be maximised, pooled where possible, and risk assessment crossvalidated among patient cohorts. Risk scores are a useful research tool and can provide clinical guidance; however, they are not yet ready to replace expert clinical judgement. There is an urgent need to identify patients that fail on current therapeutic strategies and determine how to better improve outcomes.

Future research will focus on developing a dynamic risk score from prospective interventional registry studies. We should move towards collaborative, multi-national efforts to improve sample size and data generalisability. Survival remains the ultimate measure of outcome, but good surrogates should be investigated.

\section{STATEMENT OF INTEREST}

L.J. Rubin has served on a scientific advisory committee for United Therapeutics, and has served as a consultant to NHLBI, Actelion, Pfizer, United Therapeutics, Lung LLC, Gilead, Aires Pharmaceuticals, GSK, Bayer, GeNO, Cytokinetics and Mondobiotech. G. Simonneau has received fees for speaking, educational programmes and consulting from Actelion, GSK, Pfizer, Lilly and Novartis. He has received funds for research from Actelion, GSK, Pfizer, Lilly and Novartis, and has received reimbursement for attending a symposium from Actelion, GSK, Pfizer, Lilly and Novartis. D. Badesch has received grant/research support from Glaxo Wellcome, United Therapeutics/Lung Rx, Actelion/CoTherix, Encysive, Gilead/Myogen, Pfizer, Lilly/ICOS, Bayer and Novartis. He has served as a consultant to Glaxo Wellcome/GSK, Actelion/CoTherix, Berlex, Gilead/Myogen, Encysive, Pfizer, United Therapeutics/Lung Rx, Lilly/ICOS, MondoBiotech/MondoGEN/Biogen IDEC, Bayer, Arena, and Ikaria. He has also provided assistance to legal counsel for Actelion. $\mathrm{N}$. Galiè has been involved with steering committee activities for Eli Lilly and Co., Actelion, Pfizer, Bayer Schering, GSK and Novartis. He has been a paid lecturer, for Actelion, Eli Lilly and Co., Pfizer, Bayer Schering, and GSK. He has done contract research for Actelion, Pfizer, United Therapeutics, Bayer Schering, GSK and Novartis. M. Humbert has relationships with drug companies including Actelion, Aires, AstraZeneca, Bayer, Bristol Myers Squibb, GSK, Merck, Novartis, Nycomed, Pfizer, Stallergènes, TEVA and United Therapeutics. In addition to being investigator in trials involving these companies, relationships include consultancy service and membership of scientific advisory boards. A. Keogh has received fees for speaking and clinical trials from, and been on the advisory board for Actelion, Pfizer, Bayer, Gulead, Myogen, GSK and Novartis. J. Massaro has received fees from Pfizer Inc. for statistical consulting on this project and for attending and presenting at meetings focused around this topic. The amount of fees received is US\$5,000-10,000 (approximately $€ 4,000-8,000$ ). M. Matucci Cerinic has received fees for speaking from Pfizer, Actelion, Bristol Myers Squibb and MSD. O. Sitbon has relationships with pharmaceuticals companies including Actelion, Bayer HealthCare, GSK, Lilly, Pfizer and United Therapeutics. In addition to being investigator in trials involving these companies, relationships include consultancy service, membership of scientific advisory boards, fees for speaking, funds for research and reimbursement for attending symposia. S. Kymes has received research support from Pfizer Inc. for work examining the economic burden of pulmonary arterial hypertension. This support has been US\$50,000100,000. He has also received travel support and consulting fees of less than US\$10,000.

\section{ACKNOWLEDGEMENTS}

This article summarises the conclusions of an expert meeting held in Bologna, Italy, in February 2012. The meeting was organised by Pfizer Inc. The meeting agenda and attendance was decided by the co-chairs (L.J. Rubin, G. Simonneau and S. Kymes). All authors contributed to the content and discussion during the meeting and commented on all versions of the manuscript. Medical writing assistance was provided by S. Teal (Pfizer Ltd, Tadworth, UK).

\section{REFERENCES}

1 Humbert M, Sitbon O, Chaouat A, et al. Survival in patients with idiopathic, familial, and anorexigen-associated pulmonary arterial hypertension in the modern management era. Circulation 2010; 122: 156-163.

2 Benza RL, Miller DP, Gomberg-Maitland M, et al. Predicting survival in pulmonary arterial hypertension: insights from the Registry to Evaluate Early and Long-Term Pulmonary Arterial Hypertension Disease Management (REVEAL). Circulation 2010; 122: 164-172.

3 D'Alonzo GE, Barst RJ, Ayres SM, et al. Survival in patients with primary pulmonary hypertension. Results from a national prospective registry. Ann Intern Med 1991; 115: 343-349.

4 Humbert M, Sitbon O, Yaïci A, et al. Survival in incident and prevalent cohorts of patients with pulmonary arterial hypertension. Eur Respir J 2010; 36: 549-555.

5 Benza RL, Gomberg-Maitland M, Miller DP, et al. The REVEAL Registry risk score calculator in patients newly diagnosed with pulmonary arterial hypertension. Chest 2012; 141: 354-362.

6 Palazzini M, Leci E, Bachetti C, et al. Current era survival of pulmonary arterial hypertension (PAH) patients: comparison between clinical sub-groups. Eur Heart J 2010; 31: 21.

7 Humbert M, Yaici A, de Groote P, et al. Screening for pulmonary arterial hypertension in patients with systemic sclerosis: clinical characteristics at diagnosis and long-term survival. Arthritis Rheum 2011; 63: 3522-3530.

8 Layton G, Grieve A, Rubin LJ, et al. Short term response to sildenafil therapy for pulmonary arterial hypertension is associated with long term outcome. Am J Respir Crit Care Med 2011; 183: A5898.

9 Pittrow D, Vonk-Noordegraaf A, Vachiery J-L, et al. International, prospective registry for the documentation of first-line and maintenance therapy in patients with pulmonary hypertension (CompERA-XL): aims, design, methods, and baseline characteristics of patients. Am J Respir Crit Care Med 2010; 181: A4806.

10 Rich S, Dantzker DR, Ayres SM, et al. Primary pulmonary hypertension: a national prospective study. Ann Intern Med 1987; 107: 216-223.

11 Champion H, Mathier M, Maurey J, et al. Relationship between baseline pulmonary capillary wedge pressure and response to sildenafil treatment in pulmonary arterial hypertension. Am J Respir Crit Care Med 2012; 185: A4792.

12 Liu D, Liu Q-Q, Eyries M, et al. Molecular genetics and clinical features of Chinese idiopathic and heritable pulmonary arterial hypertension patients. Eur Respir J 2012; 39: 597-603. 
13 McGoon M, Benza RL, Frost AE, et al. Validation of the French pulmonary hypertension network equation to estimate survival: a REVEAL analysis. Am J Respir Crit Care Med 2012; 185: A3828.

14 Nickel N, Golpon H, Greer M, et al. The prognostic impact of follow-up assessments in patients with idiopathic pulmonary arterial hypertension. Eur Respir J 2012; 39: 589-596.

15 Galiè N, Hoeper MM, Humbert M, et al. Guidelines for the diagnosis and treatment of pulmonary hypertension. Eur Respir J 2009; 34: 1219-1263.

16 Prospective longitudinal study of patients with idiopathic pulmonary arterial hypertension, family or taking anorectics (EFORT). NCT01185730. www.clinicaltrials.gov/ct2/show/NCT01185730 Date last accessed: May 2012. Date last updated: July 1, 2012.

17 Simonneau G, Rubin LJ, Galiè N, et al. Addition of sildenafil to long-term intravenous epoprostenol therapy in patients with pulmonary arterial hypertension: a randomized trial. Ann Intern Med 2008; 149: 521-530.

18 A study of first-line ambrisentan and tadalafil combination therapy in subjects with pulmonary arterial hypertension $(\mathrm{PAH})$ (AMBITION). NCT01178073. www.clinicaltrials.gov/ct2/show/ NCT01178073 Date last accessed: May 2012. Date last updated: December 8, 2011.

19 Effects of the combination of bosentan and sildenafil versus sildenafil monotherapy on pulmonary arterial hypertension (PAH) (COMPASS-2 study). NCT00303459. www.clinicaltrials.gov/ct2/ show/NCT00303459 Date last accessed: May 2012. Date last updated: February 17, 2012.
20 Assess the efficacy and safety of sildenafil when added to bosentan in the treatment of pulmonary arterial hypertension. NCT00323297. www.clinicaltrials.gov/ct2/show/NCT00323297 Date last accessed: May 2012. Date last updated: June 15, 2012.

21 Sitbon O, Jais X, Savale L, et al. Upfront triple combination therapy of $i . v$. epoprostenol with oral bosentan and sildenafil in idiopathic and heritable pulmonary arterial hypertension. Am J Respir Crit Care Med 2011; 183: A5910.

22 Drummond MF, Sculpher MJ, Torrance GW, et al. eds. Methods for the Economic Evaluation of Health Care Programmes. 3rd Edn. Oxford, Oxford University Press, 2005.

23 Chen YF, Jowett S, Barton P, et al. Clinical and cost-effectiveness of epoprostenol, iloprost, bosentan, sitaxentan and sildenafil for pulmonary arterial hypertension within their licensed indications: a systematic review and economic evaluation. Health Technol Assess 2009; 13: 1-320.

24 McKenna SP, Doughty N, Meads DM, et al. The Cambridge Pulmonary Hypertension Outcome Review (CAMPHOR): a measure of health-related quality of life and quality of life for patients with pulmonary hypertension. Qual Life Res 2006; 15: 103-115.

25 Murawski M, Frantz R, Gomberg-Maitland M, et al. Establishing the need for development of a patient reported outcome instrument in pulmonary arterial hypertension. Chest 2011; 140: A711.

26 Chen H, Gold W, De Marco T, et al. Development of a new patientreported outcome measure to estimate functional capacity in patients with pulmonary arterial hypertension. Am J Respir Crit Care Med 2012; 185: A3833. 